\title{
Life skills as predictors of engagement in health risk behaviours: A survey of secondary school learners
}

\author{
H. PHARAOH， J. FRANTZ AND M. SMITH
}

Department of Physiotherapy, University of the Western Cape, Private Bag X17, Bellville, 7535, Cape Town, South Africa.Email: hpharaoh@uwc.ac.za

\begin{abstract}
There is consensus that education on the prevention of health risk behaviour and an increased repertoire of life skills could facilitate a reduction in health risk behaviours. This article reports on the results of a survey among 1027 grades 8-10 learners aged 13-18 years and profiles their engagement in smoking, drug use, drinking and sexual activity. It reports on the hypothesis testing for the relationship between life skills, as measured by the Life Effectiveness Questionnaire (LEQ), and engagement in the above mentioned health risk behaviours, as measured by the Youth Risk Behaviour Surveillance Survey (YRBSS). The results concur with South African national surveys of youth risk behaviour regarding the nature and extent of engagement in health risk behaviours. Regression analyses indicated that the combination of the LEQ`s life skill domains (Time management, Achievement, Emotional control, Social competence, Active initiative, Self confidence, Intellectual flexibility and Task leadership) significantly explained between $25 \%$ and $56 \%$ of the variance in the health risk behaviours (smoking, drinking, drug use, sexual activity). Similarly, this combination of LEQ`s life skill domains significantly explained $4.1 \%$ of the variance in physical activity. Time management significantly predicted sexual activity and drug use. Achievement and emotional control significantly predicted drinking, emotional control, time management, social competence, and initiative significantly predicted smoking. Thus, it is recommended that the abovementioned life skill domains be incorporated into intervention programmes or life orientation curricula in order to reduce the incidence of health risk behaviours among South African youth.
\end{abstract}

Keywords: Health risk behaviour, life skills, smoking, drinking sexual activity, drug use, secondary school learners.

\section{How to cite this article:}

Pharaoh, H., Frantz, J. \& Smith, M. (2011). Life skills as predictors of engagement in health risk behaviours: A survey of secondary school learners. African Journal for Physical, Health Education, Recreation and Dance, June (Supplement), 70-81.

\section{Introduction}

The World Health Organization (WHO) defined life skills as a group of psychosocial competencies and interpersonal skills that help people make informed decisions and deal effectively with the demands and challenges of everyday life (WHO, 2003). Life skills are an effective tool for empowering young people to make informed and responsible decisions about their own wellbeing (Hawkins et al., 1999), and may be directed toward personal actions or 
actions toward others, as well as toward actions to change the surrounding environment in order to make it conducive to health (WHO, 2003).

Empirical evidence exists for the hypothesized beneficial impact of life skills in reducing health risk behaviour. For example, Hawkins et al. (1999) reported that a package of interventions with teachers, parents, and children provided throughout elementary grades could have an enduring effect in reducing violent behaviour, heavy drinking and sexual intercourse by age 18 years among multiethnic urban children. They further asserted that providing the youth with necessary communication, negotiation and other life skills in conjunction with targeting key contextual factors influencing youth behaviour could be used to reduce or prevent high-risk behaviour. In this way the youth would develop the confidence to engage in creative problem-solving in order to overcome social and economic barriers to self-development. These reports on combinations of life skills tested in intervention programmes and conceptualized in theoretical or conceptual papers underscore the fact that education and an increased repertoire of life skills could moderate engagement in health risk behaviours. Carelse (1994) stated that having education alone as part of intervention programmes does not necessarily change high-risk behaviour. It is of utmost importance that young people learn appropriate skills in order to put the knowledge gained into practice. Thus, compromised capacity in terms of life skills could contribute to or result in increased engagement in health risk behaviours. Creating a platform for behavioural change to occur ultimately means being able to persistently commit to meet the diverse and changing needs of individuals, as well as incorporating the characteristics of their social, cultural and physical environments that place them at risk.

Current research indicates that although every effort is being made to educate every human being on the prevention of health risk behaviour, behavioural change is not taking place effectively to combat health risk behaviour (Zulkifli \& Wong, 2002). The South African Youth Risk Behaviour Survey indicated that health risk behaviours are increasingly practised by South African youth as evidenced by increased estimates in the lifetime and current engagement from the 2002 survey (Reddy et.al. 2010). For example, alcohol use increased from $32 \%$ to $35 \%$ and cannabis use from $9 \%$ to $10 \%$. These authors also reported unchanged estimates in current use or engagement in health risk behaviours among youth despite major resources channelled toward education and interventions. For example, smoking remained unchanged at $21 \%$. Sexual activity had a marginal reduction in lifetime estimates from $41 \%$ to $38 \%$, but a major reduction was reported in those who admitted engaging in sexual activity during the last three months ( $70 \%$ to $52 \%)$. Insufficient or no physical activity increased from $38 \%$ to $42 \%$. 
Young people often engage in health risk behaviour seemingly without any regard for its consequences due to the fact that they do not see themselves as being at risk. Most theoretical models of health and risk behaviour see the individuals' judgments about risk as a fundamental element in determining behaviour (National Research Council and Institute of Medicine, 2001). The individual's beliefs about the consequences of their actions and perceptions of their vulnerability to these consequences have been conceptualized as playing a fundamental role in the effectiveness of behavioural intervention programmes. Thus attempts should be made to get those affected to recognise and acknowledge their own vulnerability to negative outcomes. Hodne (1995) and Gittler,Quigley-Rick and Saks (1990) stated that the ability to judge risks is considered an essential element of decision-making competence related to engagement in health risk behaviour. Therefore there is a need for an empirical investigation of life skills as a significant predictor of engagement in health risk behaviours among the youth. This article reports on a survey of secondary school learners in grades 8-10 and profiles their engagement in smoking, drug use, drinking and sexual activity. It analyses the hypothesis which proposes a relationship between life skills, as measured by the Life Effectiveness Questionnaire (LEQ), and engagement in the abovementioned health risk behaviours, as measured by the Youth Risk Behaviour Surveillance Survey (2002).

\section{Methodology}

The ten secondary schools in the town of Paarl, South Africa were invited to participate in a cross-sectional survey using two standardized instruments. Four accepted the invitation, two declined and four did not respond. The sample of 1027 learners were selected on the criteria of all those who had parental consent, learner assent, who were enrolled in grade 8 to 10 and were present on the day of the study.

\section{Instrument}

The LEQ was used as a measure of life skill domains as independent variables. The questionnaire measures the extent to which a person succeeds in managing his or her life using generic life skills (Neill, Marsh \& Richards, 2003). The instrument has eight domains: Time management, Social competence, Achievement, Intellectual flexibility, Task leadership, Emotional control, Initiative, and Self-confidence.

The Youth Risk Behaviour Surveillance Survey (YRBSS) measures the number of health risk behaviours that youth engage in and the extent thereof (Centre for Disease Control (CDC), 2002). This measure has been used with good success in 
South Africa in the National Youth Risk Behaviour Survey, suggesting that it is appropriate for use with this sample (Reddy et.al, 2008). For the purpose of this study, only learners' smoking, drug use, drinking and sexual activity and the extent to which learners engage in each are reported on. Both questionnaires reported acceptable reliability with internal consistency ranging from 0.51 to 0.88 indicating that the measures are appropriate for use in research (CDC, 2002; Neill, Marsh \& Richards, 2003).

The questionnaires were administered in class by either the researcher or educators who were trained in the administration of the protocol. The face-toface administration of the instrument allowed learners to ask questions for clarification and reduced errors resulting from respondents not understanding the instructions or experiencing difficulty with the language of the questionnaires.

\section{Analysis}

Descriptive statistics, specifically frequencies and percentages were used to summarise demographic data and engagement in risk behaviours. Multiple regression analysis was used to assess whether life skills significantly predict engagement in health risk behaviours. The predictors were entered simultaneously to allow them to compete with one another within the regression analyses. Five models were tested in which alcohol drinking, smoking, drug use, sexual activity and physical inactivity as the dependent variable. In each model time management, social competence, achievement, intellectual flexibility, task leadership, emotional control, active initiative and self confidence (as life skill domains) were used as the predictor or independent variables.

\section{Results}

The learners who participated in this study ranged from 13-18 years with the majority falling into the age groups of $14(35.1 \%)$ and $15(29.6 \%)$ years. More males participated in the study than females (591 and 425 respectively). The grade 8 learners (431) made up the majority of participants with grade 10 learners (238) being the fewest.

The participants also reported participating health risk behaviours. Six hundred and sixty (64.3\%) participants smoked, 510 (49.65\%) drank alcohol, 251 (24.4\%) have used dagga, $26(2.5 \%)$ have used cocaine, $258(25.1 \%)$ were sexually active and 398 (38.8\%) were physically inactive. In addition, 132 (46.8\%) participants had had one sexual partner in the past three months, 54\% reported having multiple sexual partners, $20 \%$ reported having more than four sexual partners in the last three months and $13 \%$ had more than six sexual partners. Current sexual activity, i.e. in the last three months, was reported by 
199 participants (19.37\%). Of the participants, only 311 participants responded to the question of condom use during their last sexual encounter. One hundred and eighty-four (59\%) reportedly used condoms while $127(41 \%)$ did not use a condoms. Furthermore, 50 (21.6\%) learners consumed alcohol and/ or drugs before their last sexual encounter.

Table 1 summarizes information about the engagement of the participants in smoking, drinking, drug use and sexual activity.

Table 1: Engagement in health risk behaviours $(n=1027)$

\begin{tabular}{lllll}
\hline Health risk behaviours & Responses & Frequency & $\%$ & Cum \% \\
\hline Smoking & Yes & 660 & 64.3 & 64.3 \\
& No & 353 & 34.4 & 96.7 \\
Drinking & Undecided & 14 & 1.3 & 100.0 \\
& Yes & 501 & 98.8 & 48.80 \\
Drug use: Daga & No & 510 & 49.65 & 98.45 \\
& Undecided & 16 & 1.55 & 100.0 \\
& Yes & 251 & 24.4 & 24.4 \\
Cocaine & No & 741 & 72.20 & 96.6 \\
& Undecided & 35 & 3.40 & 100.0 \\
Sexual activity & Yes & 26 & 2.5 & 2.50 \\
& No & 971 & 94.50 & 97.0 \\
& Undecided & 30 & 3.00 & 100.0 \\
Physical activity & Yes & 258 & 25.1 & 25.1 \\
& No & 730 & 71.1 & 96.2 \\
& Undecided & 39 & 3.8 & 100.0 \\
& Yes & 565 & 55.0 & 55.0 \\
& No & 398 & 38.8 & 93.8 \\
& Undecided & 64 & 6.2 & 100.0 \\
\hline
\end{tabular}

$\%=$ Percentage; Cum $\%=$ Cumulative percentage

LEQ scores for each domain could range from a minimum of three points to a maximum of 24 points. Mean scores obtained on the LEQ, as a measure of perceived competence in eight life skills domains, provided the following picture of the sample in the study: The highest mean scores were obtained on perceived self-confidence $($ Mean $=20.24 \pm 4.2)$ and achievement $($ Mean $=19.43 \pm 4.5)$. The lowest mean scores were obtained on time management (Mean $=15.55 \pm$ 
4.2) and emotional control $($ Mean $=15.59 \pm 5.02)$. The remaining domains centered around 16 scaled points: social competence (Mean $=16.03 \pm 4.4$ ); intellectual flexibility (Mean $=16.6 \pm 4.5)$; task leadership $($ Mean $=16.57 \pm 4.5)$; active initiative $($ Mean $=17.49 \pm 4.9)$.

Regression analyses: The five models testing the impact of life skills on engagement in smoking, drinking, drug use, sexual activity and physical activity respectively were all found to be significant $(\mathrm{p}=.000)$. The respective contribution of each model is reflected in Table 2.

Table 2: Error variance explained in regression models

\begin{tabular}{llll}
\hline Model & Variables & $\mathrm{R}$ & $\mathrm{R}^{2}$ \\
\hline 1 & Smoking & 0.12 & 0.35 \\
2 & Drinking & 0.11 & 0.34 \\
3 & Drug use & 0.31 & 0.56 \\
4 & Sexual activity & 0.14 & 0.38 \\
5 & Physical activity & 0.02 & 0.04 \\
\hline
\end{tabular}

From Table 3 it becomes evident that Model 1 accounted for $35 \%$ of the variance in smoking and model 2 accounted for $34 \%$ of the variance in drinking. Model 3 accounted for $56 \%$ of the variance in drug use. Model 4 accounted for $38 \%$ of the variance in sexual activity. Model 5 accounted for $4 \%$ of the variance in physical activity.

Model 1- Smoking: Emotional control $(\mathrm{p}=.000)$, Time Management $(\mathrm{p}=$ $.000)$, Social competence $(p=.037)$, and Initiative $(p=.043)$ were significant predictors of smoking controlling for intellectual flexibility, task leadership, achievement and self-confidence.

Emotional control: For every one unit increase in emotional control, there was a corresponding change in smoking habit $(b=0.141)$. A positive beta value found indicated that increases in emotional control scores correspond to increases in the smoking score. As smoking habit was coded with higher values and lower values indicating non-smoking, the positive signage on the beta actually reflects an inverse relationship rather than a positive relationship. That is, learners who had low emotional control were significantly more likely to smoke when the other life skills were controlled. 
Time management: For every one unit increase in time management there was a corresponding change in smoking $(b=.121)$. The signage on the beta is positive indicating that increases in time management scores corresponds to an increase in the smoking score. Similarly, increased time management skills significantly predicted non-smoking while controlling for the other seven life skills domains.

Social Competence: For every one unit increase in social competence, there was a corresponding change in smoking $(b=-0.097)$. The negative beta value indicates an inverse relationship between social competence and smoking. That is, increased social competence significantly predicts abstinence from smoking whereas decreased social competence significantly predicts smoking, when controlling for the other seven life skills domains were held constant. The degree of personal confidence and self-perceived ability in social interactions a learner possesses significantly predicts the likelihood that the learner will engage in smoking.

Active Initiative: For every increase in initiative, there was a corresponding change in smoking $(b=-0.089)$. The negative beta value indicates an inverse relationship between Active initiative and smoking. That is, increased initiative significantly predicts non-smoking, controlling for the other seven life skills domains.

Model 2 - Drinking: Achievement $(p=.046)$ and Emotional control $(p=.000)$ were significant predictors of Drinking controlling for the remaining life skill domains. For every one unit increase in Achievement, there was a corresponding change in drinking $(b=-0.102)$. The negative beta value indicates an inverse relationship between achievement and drinking. Increased Achievement significantly predicts abstinence from drinking, controlling for the remaining life skills domains.

Emotional Control: For every one unit increase in emotional control, there was a corresponding change in drinking $(b=-0.136)$. The negative beta coefficient indicates an inverse relationship between Emotional control and drinking. Therefore increased emotional control significantly predicts abstinence from drinking controlling for the remaining life skills domains.

Model 3 - Drug Use: Time Management was a significant predictor of Drug use $(\mathrm{p}=.004)$ controlling for the remaining life skill domains. For every increase in Time management there was a corresponding change in Drug use $(b=.121)$. The signage on the beta is positive indicating that an increase in time management score corresponds to a concomitant increase in the learners' drug use score. Higher values on Drug use indicate use and lower values indicate abstinence. Thus, the positive signage on the beta reflects an inverse relationship. 
That is, an increased time management skill significantly predicts non-drug use, controlling for the remaining life skills domains.

Model 4 - Sexual activity: Time management was a significant predictor of sexual activity $(\mathrm{p}=.023)$, controlling for the remaining life skills domains. For every increase in time management there was a corresponding change in sexual activity $(b=.098)$. The signage on the beta is positive indicating that increases in time management scores correspond to increases in the sexual activity score. Sexual activity was coded as a categorical variable with the higher value indicating sexual activity and the lower value abstinence. Thus, the positive signage on the beta coefficient actually reflects an inverse relationship. Increased time management skills significantly predict abstinence from sex, controlling for the remaining life skills domains.

Model 5 - Physical activity: Active initiative was a significant predictor for physical activity $(\mathrm{p}=.000)$. For every increase in active initiative, there was a corresponding change in smoking $(b=0.166)$. The signage on the beta indicates a positive relationship between active initiative and physical activity. That is, increased Initiative significantly predicts physical activity, controlling for the other seven life skills domains.

\section{Discussion}

The study was designed to conduct a survey among grade 8-10 learners profiling their engagement in smoking, drug use, drinking and sexual activity. It tested the hypothesis which indicated that there was a relationship between life skills, as measured by the Life Effectiveness Questionnaire, and engagement in the abovementioned health risk behaviors, as measured by the Youth Risk Behaviour Surveillance Survey. The results showed that learners were actively smoking $(64.3 \%)$ and drinking (49.65\%). A quarter of this sample used dagga (24.4\%). These findings shows alarming increases compared to results from the 2008 South African Youth Risk Behaviour Survey (Reddy et.al 2010) which indicated that $21 \%$ actively smoked and $10 \%$ used "dagga". Trends in alcohol use were similar despite efforts being made to educate the population on the prevention of health risk behaviours and the harmful effects of continued alcohol use. Those who were sexually active showed a decrease of $12.9 \%$ as the 2008 results indicated that $38 \%$ were sexually active compared to the $25.1 \%$ in the current study.

The results pertaining to current use or engagement (last 30 days) showed that learners were actively smoking (36.2\%), drinking (49.7\%), and using "dagga" $(14.8 \%)$. These findings were consistently higher than estimates from the South 
African Youth Risk Behaviour Survey of 2008. Smoking increased by $15.2 \%$, drinking increased by $14.7 \%$ and dagga use by $4.8 \%$.

Sexual activity showed a marked decrease of $32 \%$ from 2008 (52\%) compared to the current study (19.4\%). This reduction was larger than the $20 \%$ decline noted between the 2002 and 2008 surveys. Those who were physically inactive $38.8 \%$, showed a decrease compared to the 2008 estimate of $42 \%$.

The results also indicated that 50 learners $(21.6 \%)$ consumed alcohol and/ or drugs before their last sexual encounter. This finding showed an increase compared to the results reported by Reddy et.al (2010) where $14 \%$ of their participants consumed alcohol or drugs before sexual intercourse. Though this finding does not imply a causal relationship between substance use and sexual activity it is worrying that substance use is occurring prior to sex given that drug or alcohol use impairs decision-making. This raises concerns about the learners decisions to consent to sex, engagement in safe sex practices e.g. condom use, and risk reduction. The learners were also prone to other health risk behaviours that include the use of tobacco, alcohol and other substances, unprotected sexual activity, poor dietary habits, physical inactivity, and other behaviours that contribute to unintentional injuries and violence. More and more evidence shows that several of these risk behaviours tend to co-occur (Wiefferink et.al, 2006; Driskell, Dyment, Mauriello, Castle \& Sherman, 2008 ;) and have similar determinants (Wiefferink et.al, 2006; Peters et.al, 2009), which opens up opportunities for integrative programmes that address such multiple behaviours (Prochaska, 2008). Integrative programmes that address multiple risk behaviours effectively and efficiently may reduce the burden on schools and teachers (Ten Dam, 2002).

Regression analyses indicated that life skills were important in reducing or preventing health risk behaviours among grades 8-10 learners. In particular, the combination of time management, social competence, achievement motivation, intellectual flexibility, task leadership, emotional control, active initiative and self-confidence significantly explained the variance in smoking, drug use, drinking and sexual activity. This finding is consistent with results of studies reported in the literature indicating that life skills training could reduce engagement in health risk behaviours (Hawkins et al., 1999).

Smoking was significantly predicted by 1) the extent to which a learner perceives him or herself to maintain emotional control when faced with potentially stressful situations, and 2) the extent to which a learner perceives that they make optimal use of time. In other words, increased perceptions of effective emotional regulation under stress (emotional control) and increased personal 
confidence and perceived social efficacy (social competence) could significantly predict non-smoking behaviour and vice versa.

Alcohol drinking was significantly predicted by 1) the extent to which a learner likes to initiate action in new situations, 2) the extent to which a learner is motivated to achieve excellence and put the required effort into it, and 3) the extent to which a learner perceives him or herself to maintain emotional control when faced with potentially stressful situations. That is, increased capacity for active initiative-taking in new situations and increased motivation and effort to achieve significantly predicts abstinence from drinking. Similarly, increased perceptions of effective emotional regulation under stress can significantly predict abstinence from alcohol.

Time management emerged fairly consistently as a significant predictor across three regression models suggesting that the extent to which a learner perceives that they make optimal use of time should be highlighted in life orientation curricula or other school-based intervention programmes. Physical activity was significantly predicted by the extent to which a learner likes to initiate action in new situations. That is, increased capacity for active initiative-taking in new situations and increased motivation and effort to be successful significantly predicts increased physical activity. Such a finding is consistent with reports in the literature (Jarvie \& Maguire, 1994; Sallis, Prochaska \& Taylor, 2000).

\section{Conclusion and Recommendations}

Learners who participated in this survey showed an alarming degree of engagement in health risk behaviours. This engagement raises major concerns as every effort is being made to educate everyone especially the youth on the harmful effects of health risk behaviours. Despite all the efforts being made learners are putting themselves at risk with no regard for the consequences of their actions. The results support that the combination of the eight life skills domains, measured by the LEQ, as significant predictors of engagement in smoking, drinking, drug use, sexual activity and physical activity. It is recommended that the above mentioned life skill domains be incorporated into intervention programmes or life orientation curricula, and that the significant predictors be underscored in the content of intervention programmes in order to assist in the prevention of health risk behaviours.

\section{References}

Carelse, M. (1994). An investigation into sexual behavior and knowledge of AIDS at an industrial school for boys. Unpublished Doctoral dissertation, University of the Western Cape, Department of Psychology, Cape Town. 
Driskell, M.M., Dyment, S., Mauriello, L., Castle, P. \& Sherman, K. (2008). Relationships among multiple behaviors for childhood and adolescent obesity prevention. Preventative Medicine, 46, 209-215.

Gittler, J., Quigley-Rick, M. \& Saks, M.J. (1990). Adolescent Health Care Decision Making: The Law and Public Policy. Carnegie Council on Adolescent Development, Washington, DC.

Hawkins, J.D., Catalano, R.F., Kosterman, R., Abbott, R. \& Hill, K.G. (1999). Preventing adolescent health-risk behaviors by strengthening protection during childhood. Archives of Pediatric and Adolescent Medicine, 153;226-234.

Hodne, C.J. (1995). Medical decision making. In O'Hara, M.W. \& Reiter, R.C. (Eds.), Psychological Aspects of Women's Reproductive Health. Springer, New York, pp. 267-290.

National Research Council and Institute of Medicine. (2001). Adolescent

Risk and Vulnerability: Concepts and Measurement. Board on Children, Youth, and Families. Division of Behavioral and Social Sciences and Education. Baruch Fischhoff. In Elena O. Nightingale \& Joah G. Iannotta (Eds.). Washington, DC: National Academy Press.

Neill, J. T., Marsh, H. W. \& Richards, G. E. (2003). The Life Effectiveness Questionnaire: Development and psychometrics. Unpublished manuscript, University of Western Sydney, Sydney, NSW, Australia.

Peters, L.W.H. et al. (2009). A review of similarities between domain specific determinants of four health behaviors among adolescents. Health Education Research, 24, 198-223.

Prochaska, J.O. (2008). Multiple health behavior research represents the future of preventive medicine. Preventative Medicine, 46, 281-285.

Reddy, S.P., James, S., Sewpaul, R., Koopman, F., Funani, N.I., Sifunda, S., Josie, J., Masuka, P., Kambaran, N.S. \& Omardien, R.G. (2010). Umthente Uhlaba Usamila - The South African Youth Risk Behaviour Survey 2008. Cape Town: South African Medical Research Council.

Sallis J.F., Prochaska J.J. \& Taylor W.C. (2000). A review of correlates of physical activity of children and adolescents. Medicne Science Sports \& Exercise, 32, 963-975.

Ten Dam GTM (2002). Effectiveness in health education. In Conference Report: Education and Health in Partnership: A European Conference on Linking Education with the Promotion of Health in Schools: 25-27 Septem-ber 2002; Egmond aan Zee, The Netherlands T.Young Woerden (Eds.). The Netherlands: Netherlands Institute for Health Promotion and Disease Prevention/International Planning Committee of the European Network of Health Promoting Schools; 17-22.

Wiefferink, C.H., Peters, L., Hoekstra, F., Ten Dam, G., Buijs, G.J. \& Paulussen, T.G.W.M. (2006). Clustering of health-related behaviors and their determinants: possible consequences for school health interventions. Preventative Science, 7, 127-149.

World Health Organization, Regional Office for the Western Pacific (WHO/WPRO) (2003). Value Adolescents, Invest in Future: Educational Package. Facilitator's Manual. Manila, Philippines. 
YRBSS: Youth Risk Behavioural Surveillance System 2002 (On Line). Retrieved February, 09, 2011 at 11:26 from www.cdc.gov/mmwr/preview/mmwrhtml/ss5104a1.htm

Zulkifli, S.N. \& Wong, Y. L. (2002). Knowledge, attitudes and beliefs related to HIV/AIDS among adolescents in Malaysia.The Medical Journal of Malaysia, 57(1), 3-23. 